\title{
Study on the antibacterial activities of emodin derivatives against clinical drug-resistant bacterial strains and their interaction with proteins
}

\author{
Chengjie Ji, Guang Xin ${ }^{2}$, Feixia Duan ${ }^{3}$, Wen Huang ${ }^{2}$, Taichang Tan ${ }^{1}$ \\ ${ }^{1}$ Department of Laboratory Medicine, Sichuan Provincial People's Hospital, University of Electronic Science and Technology of China, Chengdu \\ 611731, China; ${ }^{2}$ Laboratory of Ethnopharmacology, Regenerative Medicine Research Center, Institute for Nanobiomedical Technology and \\ Membrane Biology, West China Hospital/West China Medical School, Sichuan University, Chengdu 610041, China; ${ }^{3}$ College of Light Industry, \\ Textile and Food Engineering, Sichuan University, Chengdu, Sichuan 610065, China \\ Contributions: (I) Conception and design: C Ji, W Huang; (II) Administrative support: T Tai, W Huang; (III) Provision of study materials: G Xin; (IV) \\ Collection and assembly of data: C Ji, F Duan; (V) Data analysis and interpretation: C Ji, F Duan; (VI) Manuscript writing: All authors; (VII) Final \\ approval of manuscript: All authors. \\ Correspondence to: Wen Huang. Laboratory of Ethnopharmacology, Regenerative Medicine Research Center, Institute for Nanobiomedical \\ Technology and Membrane Biology, West China Hospital/West China Medical School, Sichuan University, Keyuan 4 Road No. 1, Gaopeng Avenue, \\ Gaoxin District, Chengdu 610041, China. Email: huangwen@scu.edu.cn; Taichang Tan. Department of Laboratory Medicine, Sichuan Academy of \\ Medical Sciences and Sichuan Provincial People’s Hospital, Chengdu 610072, China. Email: sinotan@sina.com.
}

\begin{abstract}
Background: Novel haloemodin (HEI2) synthesized by modifying emodin, a traditional Chinese medicine component, possesses remarkable antibacterial activity, being much more effective than its parent nucleus, emodin.

Methods: Firstly, we discovered that HEI2 increases bacterial cell membrane permeability to potassium ions more drastically than emodin. We thus further investigated the interaction of haloemodin and protein using a fluorescence quenching and circular dichroism (CD) study based on bovine serum albumin (BSA).

Results: HEI2 spontaneously bound to BSA at Trp 212 residue (subdomain IIA) by hydrogen bonds and van der Waals interactions to forms HEI2-BSA complexes, and this binding decreased the $\alpha$-helical content of BSA. We also confirmed that emodin bound to BSA by hydrophobic interaction alone.

Conclusions: These results suggest that the main responses for the substantial antibacterial activities of HEI2 are a disruption of the bacterial plasma membrane function and the interaction with biological functional proteins. Furthermore, the study of the interaction of drugs with BSA, which has a fluorescent group tryptophan residue similar to many bio-functional proteins, will be a simple and inexpensive scopereducing method in screening new drugs.
\end{abstract}

Keywords: Haloemodin; antibacterial activity; bovine serum albumin (BSA); fluorescence quenching; circular dichroism (CD)

Submitted Nov 18, 2019. Accepted for publication Dec 11, 2019.

doi: $10.21037 /$ atm.2019.12.100

View this article at: http://dx.doi.org/10.21037/atm.2019.12.100

\section{Introduction}

Emodin (1,3,8-trihydroxy-6-methylanthraquinone, Figure $1 A$ ), an active anthraquinone component isolated from the root of Rheum palmatum $L$ (1), is capable of a variety of bioactivities including anti-tumor (2), antioxidant $(3,4)$, antibacterial, and vasorelaxant effects $(5,6)$. Drugs containing an anthraquinone moiety such as daunorubicin (7) and mitoxantrone (8) have been used for inhibiting various cancer cells in the clinic. However, the low efficiency and bioavailability of emodin restrict 


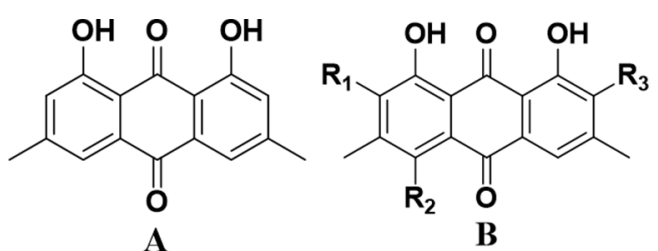

Figure 1 Chemical structures of emodin and haloemodins. (A) Emodin and (B) haloemodins. HEI1: R1 = I, R2 = R3 = H; HEI2: $\mathrm{R} 1=\mathrm{R} 2=\mathrm{I}, \mathrm{R} 3=\mathrm{H} ; \mathrm{HEI} 3: \mathrm{R} 1=\mathrm{R} 2=\mathrm{R} 3=\mathrm{I}$.

its application in clinic for curing infections, cancers, etc. We therefore synthesized a class of novel haloemodins (Figure $1 B$ ) by introducing iodine substituents on its ring, and investigated their bioactivities in vitro and in vivo. As we expected, the novel haloemodins, including 2,4-diiodoemodin (HEI2), 2-iodioemodin (HEI1), and 2,4,5-triiodoemodin (HEI3) (Figure 1B), showed significant antibacterial activities against Gram-positive bacteria even for several drug-resistant clinical bacteria like methicillinresistant Staphylococcus aureus (MRSA) and vancomycinresistant Enterococcus faecium (VRE), with least 50 times more efficiency than its parent nucleus, emodin (9).

The plasma membrane is essential for bacterial cell viability due to its barrier and transport function, while bio-macromolecules, especially proteins, play a crucial role in bacterial cell division, multiplication, metabolism and pathogenicity. Examining the binding of small molecules with proteins has great value in pharmacology, pharmacy, and biochemistry. To investigate the mechanism underlying antibacterial activity promotion by iodine substitutions on the anthraquinone ring of emodin, the plasma membrane permeability of Gram-positive bacteria, Staphylococci aureus and Bacillus cereus, as detected in culture with haloemodins, and fluorescence spectroscopy, ultraviolet-visible (UV-vis) absorption spectrum, and circular dichroism (CD) were used to study the binding mechanism, binding parameters, binding site, and other features of the haloemodin and protein.

Bovine serum albumin (BSA) is one of the most extensively investigated proteins, as it is easy to acquire, is highly stable, and displays structural homology with human serum albumin (HSA) $(10,11)$. BSA is a globular, heart-shaped protein, composed of three linearly arranged, homologous domains (I, II, and III) which are divided into 9 loop by 17 disulfide bonds (3), with each domain containing two sub-domains (A and B). From crystallographic data of X-rays, it has been revealed that the structure of the albumin in BSA is largely $\alpha$-helical with the left polypeptide occurring in turns $(7,12)$. BSA has two tryptophan residues that have intrinsic fluorescence (3). Trp-212 usually occurs in a hydrophobic binding pocket of subdomain IIA, while Trp-134 can be found on the surface of the subdomain IB. The location of the drug binding sites of BSA is usually in the hydrophobic cavities of subdomain IIA and IIIA (5). Therefore, a binding mode study of those drugs with proteins using BSA as a model protein may be a useful method for new drug screening and pharmacology research.

\section{Methods}

\section{Materials}

Albumin bovine $\mathrm{V}$ was purchased from Roche (USA), and emodin was purchased from Sigma (USA). The haloemodins including 2,4-diiodoemodin, 2-iodioemodin and 2,4,5-triiodoemodin (purity >95\%) were synthesized in our lab with the structure being thoroughly clarified using high resolution mass spectrometer (HRMS) and nuclear magnetic resonance (NMR) (9). Staphylococcus aureus ATCC6538, Bacillus cereus ATCC10231, and Enterococcus faecalis ATCC 29212 were acquired from the College of Food Science and Nutritional Engineering, China Agricultural University, and the clinical isolates were acquired from the Institute of Clinical Pharmacology, Peking University. All other reagents were of analytical grade.

\section{Preparation of stock solutions}

The BSA solution $(100 \mu \mathrm{M})$ was prepared in $50 \mathrm{mM}$ phosphate buffer with $\mathrm{pH} 7.4$ containing $100 \mathrm{mM} \mathrm{NaCl}$. A stock solution of haloemodin $(1 \mathrm{mM})$ was prepared in water. All stock solutions were stored at $4{ }^{\circ} \mathrm{C}$.

\section{Potassium measurements}

To assess the free extracellular potassium, Staphylococcus aureus ATCC6538 and Bacillus cereus ATCC10231 were loaded with sodium phosphate buffer $(50 \mathrm{mM}$, $20 \mathrm{mM} \mathrm{NaCl}$ ) and $50 \mu \mathrm{M} \mathrm{HEI} 2$ for different time-points $(20,40,60,120$, and $240 \mathrm{~min})$ at $37^{\circ} \mathrm{C}$. The potassium concentration was measured by ZA3000 atomic absorption spectrophotometer (Hitachi Ltd., Tokyo, Japan). 


\section{UV-Vis absorbance spectroscopy}

Measurements of the absorbance spectrum were conducted using a Beckman DU800 nucleic acid/protein analyzer (Beckman Coulter Ltd., CA, USA) with a quartz cell of $1 \mathrm{~cm}$ path length (13). The absorbance spectra were recorded with final BSA and a drug concentration of $50 \mu \mathrm{M}$.

\section{Fluorescence spectroscopy}

Fluorimetric experiments were conducted using a Hitachi F-7000 Fluorescence Spectrophotometer (Hitachi Ltd., Tokyo, Japan). A wavelength range of 300-500 nm was the setting for the fluorescence spectra assessment, which was performed by exciting the serum albumins at $280 \mathrm{~nm}$ using a slit width of $5 \mathrm{~nm}$.

The quenching data were calculated using the SternVolmer equation (14):

$$
\frac{F_{0}}{F}=1+K_{q} \tau_{0}[Q]=1+K_{s v}[Q]
$$

Where $\mathrm{F}_{0}$ and $\mathrm{F}$ are the fluorescence intensity in the absence and presence of a quencher, $\mathrm{Kq}$ is the biomolecular quenching rate constant, [Q] is the concentration of quencher, Ksv is Stern-Volmer dynamic quenching constant, $\tau 0$ represents the average lifetime of the protein without the quencher, and the value of $\tau 0$ is $10^{-8} \mathrm{~s}(15)$.

When small molecules interact with a set of commensurate sites of a macromolecule, the equilibrium of free and bound molecules can be calculated using the following equation (16):

$$
\log \frac{F_{0}-F}{F}=\log K+n \log [Q]
$$

Where $\mathrm{K}$ and $\mathrm{n}$ represent the binding constant and the number of binding sites per albumin molecule.

Generally, small molecular substrates bind to protein through four kinds of forces: hydrogen bond, van der Waals force, electrostatic and hydrophobic interactions (3). For the purpose of confirming the acting forces influencing the drugs-BSA interactions, the temperature-dependent thermodynamic parameters were obtained using the Van't Hoff equation (13):

$$
\ln \frac{K_{2}}{K_{1}}=\left(\frac{1}{T_{1}}-\frac{1}{T_{2}}\right) \frac{\Delta H}{R}
$$

Where $\mathrm{K} 1$ and $\mathrm{K} 2$ are the binding constant at the corresponding temperature, and $\mathrm{R}$ is the gas constant. The tests were conducted at 298 and $310 \mathrm{~K}$. The fluctuations in enthalpy and entropy of quenching process are represented below by $\Delta \mathrm{H}$ and $\Delta \mathrm{S}$, respectively .

$\Delta \mathrm{G}$ related to the interaction of haloemodin with BSA is calculated as follows:

$$
\Delta G=-R T \ln K=\Delta H-T \Delta S
$$

The transfer of energy occurs through the direct electrodynamic interaction of the primarily excited molecule and its neighbors, which occurs when the donor is able to produce fluorescent light, when the fluorescent emission spectrum of the donor and $\mathrm{UV}$-vis absorbance spectrum of the acceptor overlap, and when they are separated by less than $8 \mathrm{~nm}$ (17). The binding distance (r) between the donor fluorophore and acceptor can be found using Forster resonance energy transfer (FRET) (18), which can be calculated as follows (19):

$$
E=1-\frac{F}{F_{0}}=\frac{R_{0}^{6}}{R_{0}^{6}+r^{6}}
$$

Where $\mathrm{E}$ is the energy transfer efficiency, $\mathrm{F}$ and $\mathrm{F}_{0}$ are the fluorescence intensities of BSA in the presence and absence of drugs, $r$ is the distance between the acceptor and the donor, and $\mathrm{R}_{0}$ is the critical distance at which $50 \%$ of the excitation energy is transferred to the acceptor (20). It can be calculated as follows (21):

$$
R_{0}^{6}=8.79 \times 10^{-25} K^{2} N^{-4} \varphi J
$$

Where $\mathrm{K} 2$ is the spatial orientation factor, $\mathrm{N}$ is the refractive index of medium, $\varphi$ is the quantum yield of the donor in the absence of the acceptor, and $\mathrm{J}$ is the overlap integral of the emission spectrum of the donor and the absorption spectrum of the acceptor. $\mathrm{J}$ is calculated as follows (21):

$$
J=\frac{\int_{0}^{\infty} F(\lambda) \varepsilon(\lambda) \lambda^{4} d \lambda}{\int_{0}^{\infty} F(\lambda) d \lambda}
$$

Where $F(\lambda)$ is the fluorescence intensity of the fluorescent donor at wavelength $\lambda$, and where $\varepsilon(\lambda)$ is the molar absorption coefficient of the acceptor at wavelength $\lambda$.

\section{CD spectroscopy}

The CD spectra between 190 and $260 \mathrm{~nm}$ were recorded on an AVIV Model 400 CD spectrometer (Aviv Biomedical, Inc., Lakewood, NJ, USA) at $25^{\circ} \mathrm{C}$, using a $0.2 \mathrm{~cm}$ path length cell. Spectra were gathered at intervals of $1 \mathrm{~nm}$ with 

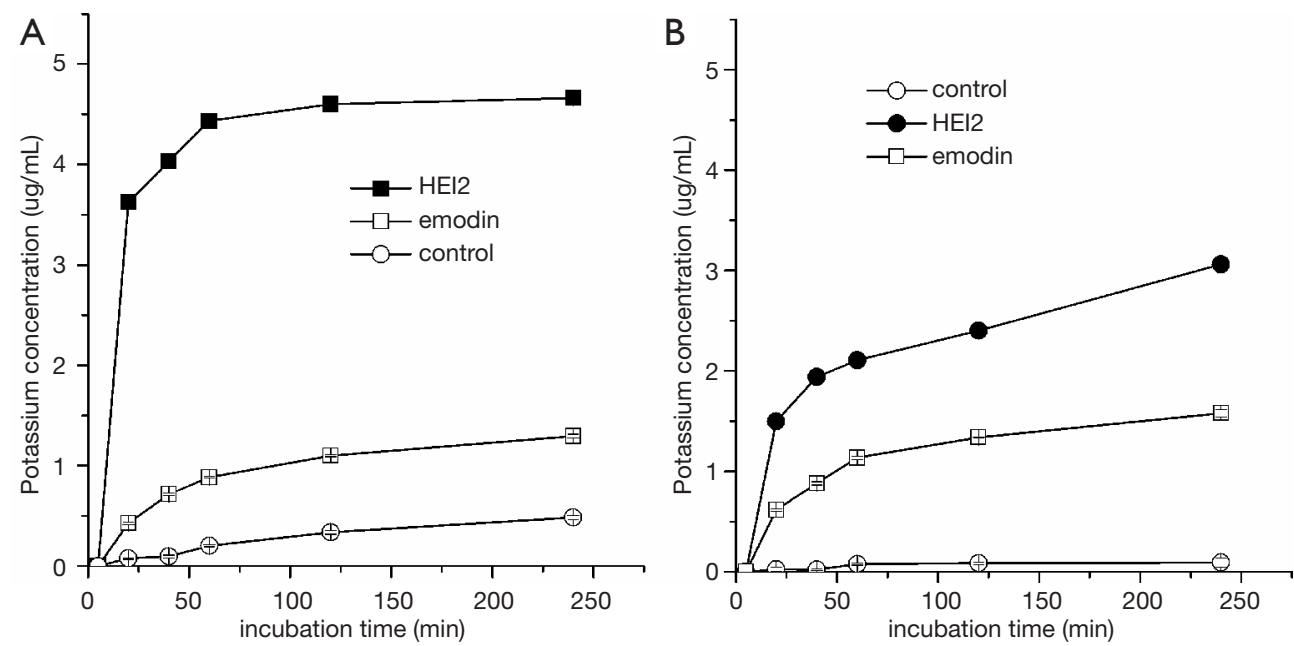

Figure 2 The extracellular potassium concentration of Staphylococcus aureus ATCC6538 (A), Bacillus cereus ATCC10231 (B) with emodin and HEI2. HEI2, 2,4-diiodoemodin.

a $1 \mathrm{~s}$ signal averaging time. Three scans were averaged, and all the data were expressed as mean residue ellipticity, $[\theta]$, in units of degrees $\cdot \mathrm{cm}^{2} \cdot \mathrm{dmol}^{-1}$.

The secondary structures of the protein were calculated by the software CDNN (Institut für Biotechnologie, Martin-Luther-Universität Halle-Wittenberg, Germany).

\section{Results}

\section{Antibacterial activity analysis}

In our previous study, we found that all three haloemodins inhibit Gram-positive bacteria growth including that of $S$. aureus, B. laterosporus, and E. faecium (9). The MIC of HEI2 for $S$. aureus ATCC 6538 was $0.002 \mathrm{mg} / \mathrm{mL}$, which is less than $1 \%$ of that of emodin $(0.256 \mathrm{mg} / \mathrm{mL})$, and the same as that of vancomycin. Furthermore, the MIC of HEI2 for MRSA and VRE was $0.004-0.032$ and $0.008-0.016 \mathrm{mg} / \mathrm{mL}$, respectively, whereas the MIC of cefoxitin for MRSA was $0.128 \mathrm{mg} / \mathrm{mL}$.

\section{HEI2 increases bacterial cell membrane permeability for $\mathrm{K}+$}

The plasma membrane is essential for bacterial cell viability due to its barrier and transport function. The potassium-ion permeability of bacterial cell plasma membrane is able to not only control the ion concentration in and out of cell, but can also alter the membrane potential and trans-membrane resting potential in turn. To reveal the effect of haloemodin on bacterial membrane permeability, the extracellular potassium concentrations of $S$. aureus ATCC6538 and $B$. cereus ATCC10231 treated with HEI2 for gradient time were determined using atomic absorption spectrometry. As shown in Figure 2, the extracellular potassium concentration of HEI2-S. aureus group rose to 3.2 and $4.5 \mu \mathrm{g} / \mathrm{mL}$ after about 20 and $60 \mathrm{~min}$ of treatment, respectively, while that of emodin-S. aureus group was 0.4 and $0.9 \mu \mathrm{g} / \mathrm{mL}$, respectively. A similar phenomenon was observed in the $B$. cereus groups. These results imply that the introduction of iodine substituents to the anthraquinone ring of emodin can increase bacterial plasma membrane permeability for potassium ions, which is an important contributory factor in the inhibition against the growth of bacterial cells of HEI2.

\section{Interaction of BSA with baloemodin}

\section{Quenching mechanism analysis}

HEI2 showed intense inhibitory effect on both bacterial topoisomerase I and DNA gyrase (9). To simulate the effect of the interaction between these enzymes with haloemodin, we chose the classical protein, BSA, in a quenching mechanism study. The fluorescence of the tryptophan residues of $\mathrm{BSA}$ was monitored, and the maximum emission wavelength was $340 \mathrm{~nm}$. The fluorescence quenching spectra of BSA with haloemodin are displayed in Figure $3 A, B, C, D$.

As is evident from Figure 3, a fluorescence intensity decrease of $340 \mathrm{~nm}$ was accompanied by an increase in haloemodin concentrations. The result revealed that 

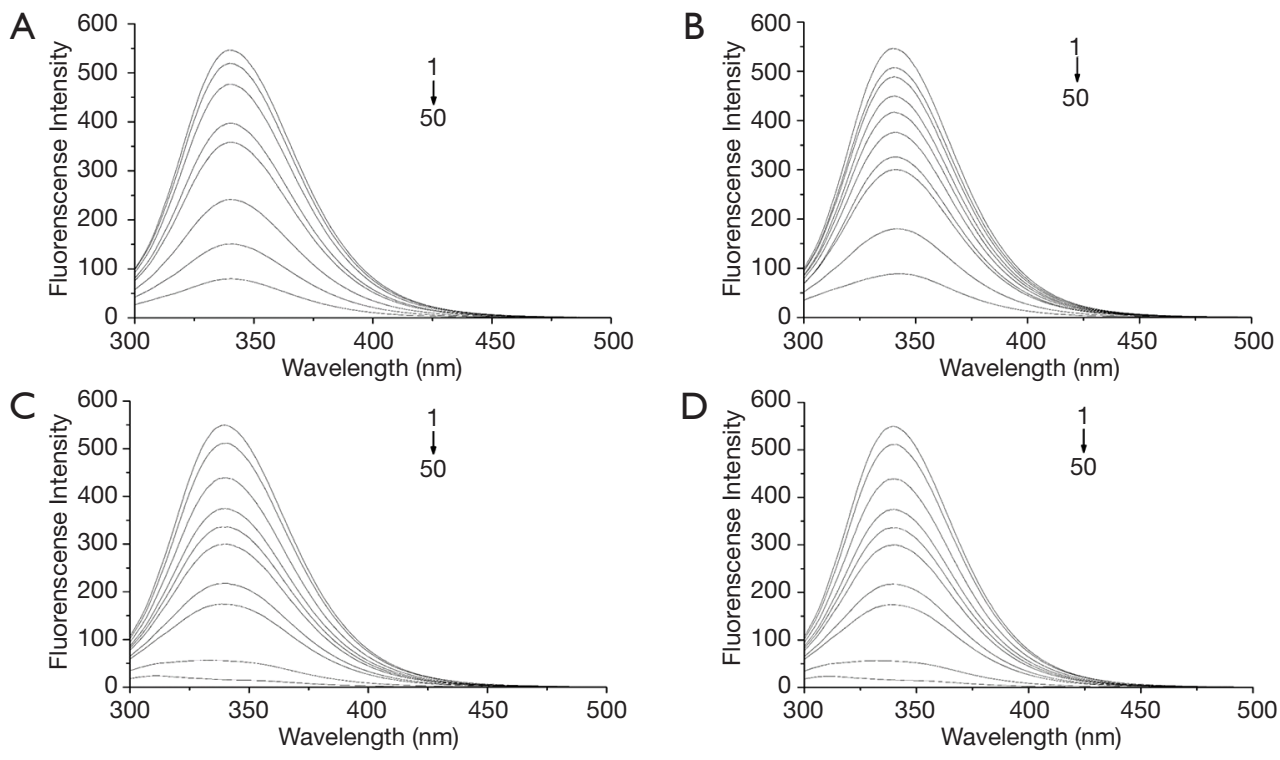

Figure 3 Fluorescence quenching spectra of BSA with emodin (A), HEI1 (B), HEI2 (C), and HEI3 (D). BSA =5 $\mu$ M, HEI1 = HEI2 $=\mathrm{HEI} 3=0,1,2,3,4,5,7.5,10,25$, and $50 \mu \mathrm{M}$. BSA, bovine serum albumin; HEI1, 2-iodioemodin; HEI2, 2,4-diiodoemodin; HEI3, 2,4,5-triiodoemodin.

haloemodin can bind to BSA and quench the fluorescence of tryptophan residues.

Dynamic quenching involves the quencher diffusing to the fluorophore within the duration of the exited state. Meanwhile, static quenching describes the development of a nonfluorescent fluorophore-quencher complex. The maximum diffusion-collision-quenching rate constant of various quenchers with a biopolymer is $2.0 \times 10^{10} \mathrm{~L} \cdot \mathrm{mol}^{-1} \cdot \mathrm{s}^{-1}(14)$. Sterm-Volmer plots for BSA with emodin derivatives are shown in Figure 4. The values of $\mathrm{Kq}$ derived from Eq. [1] is $3.16 \times 10^{12}$ and $1.02 \times 10^{13}$; meanwhile, $3.69 \times 10^{13}$ and $3.81 \times 10^{13} \mathrm{~L} \cdot \mathrm{mol}^{-1} \cdot \mathrm{s}^{-1}$ are the values for the binding of emodin, along with HEI1, HEI2, and HEI3 at $298 \mathrm{~K}$ (Table 1). This demonstrates that the quenching processes are static quenching.

The decrease of $\mathrm{Kq}$ and $\mathrm{Ksv}$ with a rising temperature also suggests that quenching should be ascribed to complex formation and not dynamic collision (22). Also, the Ksv value of haloemodin was greater than that of emodin, resulting in the haloemodin exhibiting a stronger quenching ability than emodin. The higher Ksv and $\mathrm{Kq}$ values for the HEI2-BSA system imply that HEI2 quenches BSA through complexation more effectively than emodin.

\section{Binding constant and the binding site number}

Plots of $\log \left(\mathrm{F}_{0}-\mathrm{F}\right) / \mathrm{F}$ versus $\log [\mathrm{Q}]$ are illustrated in Figure 5 , while the values of binding constant $\mathrm{K}$ and the number of binding sites per BSA are shown in Table 2. The binding capacity of emodin and haloemodin declined with increasing temperature, suggesting that emodin/haloemodin-BSA was sensitive to temperature. The values of $\mathrm{n}$ are almost equal to 1 , which shows that there was a single binding site in BSA, and the drugs were most likely bound to Trp-212 within the hydrophobic pocket of subdomain IIA. The $\mathrm{n}$ value for emodin smaller than 1 indicated that emodin was limited to being bound to BSA $(23,24)$. For HEI2-BSA, the $n$ value was bigger than 1 , which corresponded to a powerful binding force between HEI2 and BSA. The data revealed that the binding ability of HEI2 to emodin was stronger than that of BSA.

\section{Thermodynamic parameters and binding modes}

On the basis of the binding constants that were acquired at different temperatures ( 298 and $310 \mathrm{~K})$, the alteration of entropy $(\Delta S)$ and enthalpy $(\Delta \mathrm{H})$ values were found using Eq. [3], while the free energy change $(\Delta G)$ was found using Eq. [4]. The results are presented in Table 3. The importance of the thermodynamic parameters in the protein association process has been presented by Ross and Subramanian (25). The negative $\Delta G$ value indicated that the interaction process was spontaneous. Consequently, the BSA-haloemodin binding process must have been 

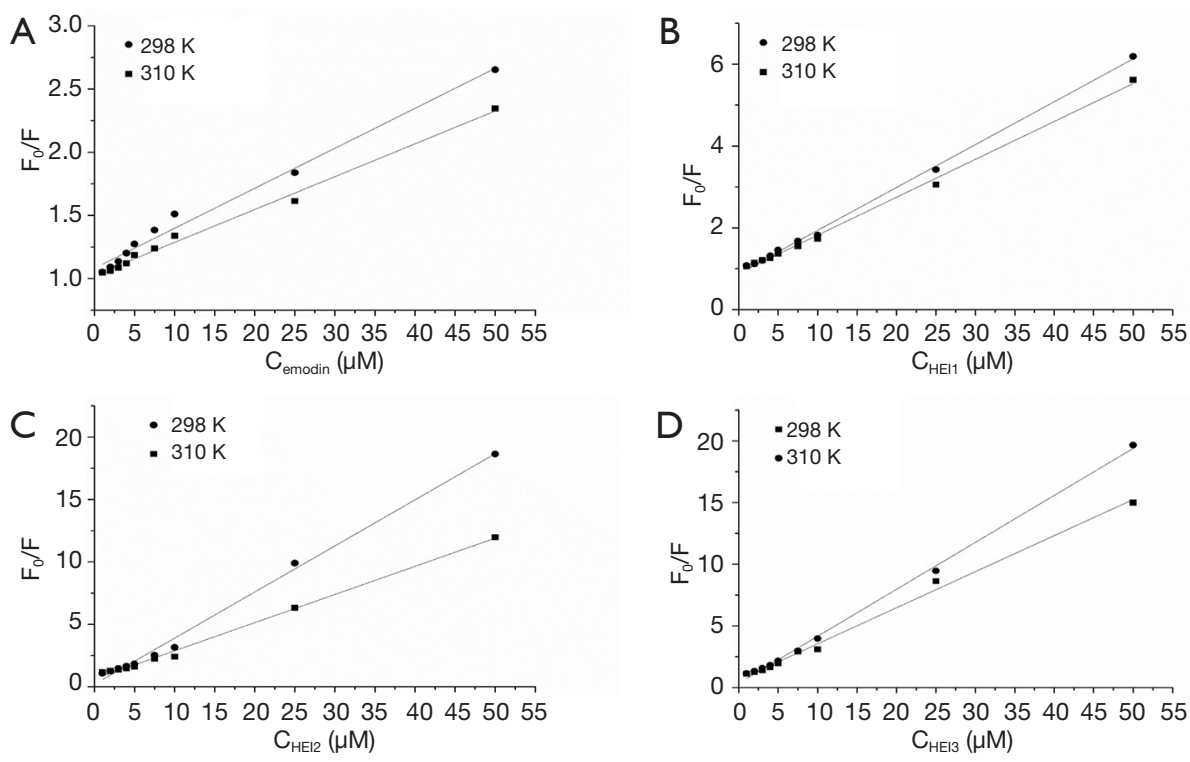

Figure 4 Sterm-Volmer plots for BSA with emodin (A), HEI1 (B), HEI2 (C) and HEI3 (D) at 298 and 310 K. BSA, bovine serum albumin; HEI1, 2-iodioemodin; HEI2, 2,4-diiodoemodin; HEI3, 2,4,5-triiodoemodin.

Table 1 Stern-Volmer quenching equation of emodin, HEI1, HEI2, and HEI3 to BSA

\begin{tabular}{lcccc}
\hline Variable & $\mathrm{T} / \mathrm{K}$ & $\mathrm{Kq}(\mathrm{L} / \mathrm{mol} / \mathrm{s})$ & $\mathrm{Ksv}(\mathrm{L} / \mathrm{mol})$ & $\mathrm{r}$ \\
\hline Emodin & 298 & $3.16 \times 10^{12}$ & $3.16 \times 10^{4}$ & 0.9933 \\
& 310 & $2.60 \times 10^{12}$ & $2.60 \times 10^{4}$ & 0.9967 \\
HEI1 & 298 & $1.02 \times 10^{13}$ & $1.02 \times 10^{5}$ & 0.9950 \\
& 310 & $9.26 \times 10^{12}$ & $9.26 \times 10^{4}$ & 0.9985 \\
HEI2 & 298 & $3.69 \times 10^{13}$ & $3.69 \times 10^{5}$ & 0.9981 \\
& 310 & $2.26 \times 10^{13}$ & $2.26 \times 10^{5}$ & 0.9975 \\
HEI3 & 298 & $3.81 \times 10^{13}$ & $3.81 \times 10^{5}$ & 0.9976 \\
& 310 & $2.93 \times 10^{13}$ & $2.93 \times 10^{5}$ & 0.9990 \\
\hline
\end{tabular}

$r$ is the correlation coefficient. HEl1, 2-iodioemodin; HEl2, 2,4-diiodoemodin; HEI3, 2,4,5-triiodoemodin; BSA, bovine serum albumin.

spontaneous. As $\Delta \mathrm{H}$ was very small (almost 0 ), its negative value cannot be accounted for by electrostatic interactions. In minor interactions between molecules and protein, a positive $\Delta S$ value is usually considered to be proof of a hydrophobic interaction as the water molecules have a more random configuration than when they are in an organized mode around the drug and protein (26). The negative values of $\Delta \mathrm{H}$ and $\Delta \mathrm{S}$ indicate that the forces between HEI2 and BSA molecules consisted mostly of hydrogen bonds and van

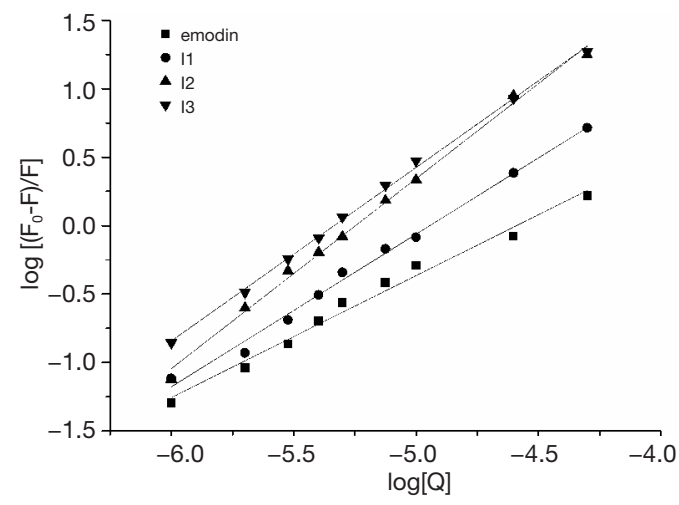

Figure 5 Double-log plots of BSA-haloemodin system. BSA, bovine serum albumin.

der Waals interactions. Furthermore, the negative $\Delta \mathrm{H}$ and positive $\Delta S$ value s suggest that hydrophobic interactions were critically involved in the binding process of emodin, HEI1, and HEI3 with BSA. The primary forces informing the emodin-BSA and HEI2-BSA interactions were different, which gave rise to varying compound stabilities; this may explain the variation in antibacterial activity.

It is also worth noting that, compared to emodin-BSA, the value of $\Delta \mathrm{H}$ for haloemodin-BSA was dramatically lower, suggesting that the modified emodin-BSA complex is more stable than emodin-BSA. 
Table 2 The $\mathrm{n}$ and $\mathrm{K}$ value of BSA with emodin, HEI1, HEI2, and HEI3

\begin{tabular}{lcccc}
\hline Variable & Emodin & HEl1 & HEI2 & HEI3 \\
\hline $\mathrm{K}$ & & & & \\
298 & $1.23 \times 10^{4}$ & $3.26 \times 10^{5}$ & $1.93 \times 10^{7}$ & $5.75 \times 10^{6}$ \\
310 & $8.67 \times 10^{3}$ & $2.45 \times 10^{5}$ & $7.87 \times 10^{5}$ & $4.88 \times 10^{6}$ \\
$\mathrm{n}$ & 0.89 & 1.10 & 1.39 & 1.27 \\
\hline
\end{tabular}

BSA, bovine serum albumin; HEl1, 2-iodioemodin; HEI2, 2,4-diiodoemodin; HEI3, 2,4,5-triiodoemodin.

Table 3 Thermodynamic parameters of the interaction between BSA and emodin, HEI1, HEI2, and HEI3

\begin{tabular}{lccc}
\hline Variable & $\Delta \mathrm{H}$ & $\Delta \mathrm{G}$ & $\Delta \mathrm{S}$ \\
\hline Emodin & -22.17 & -22.47 & 1.01 \\
HEl1 & -18.16 & -31.45 & 44.60 \\
HEI2 & -204.79 & -41.56 & -547.74 \\
HEl3 & -10.5 & -38.56 & 94.17 \\
\hline
\end{tabular}

BSA, bovine serum albumin; HEl1, 2-iodioemodin; HEI2, 2,4-diiodoemodin; HEI3, 2,4,5-triiodoemodin.
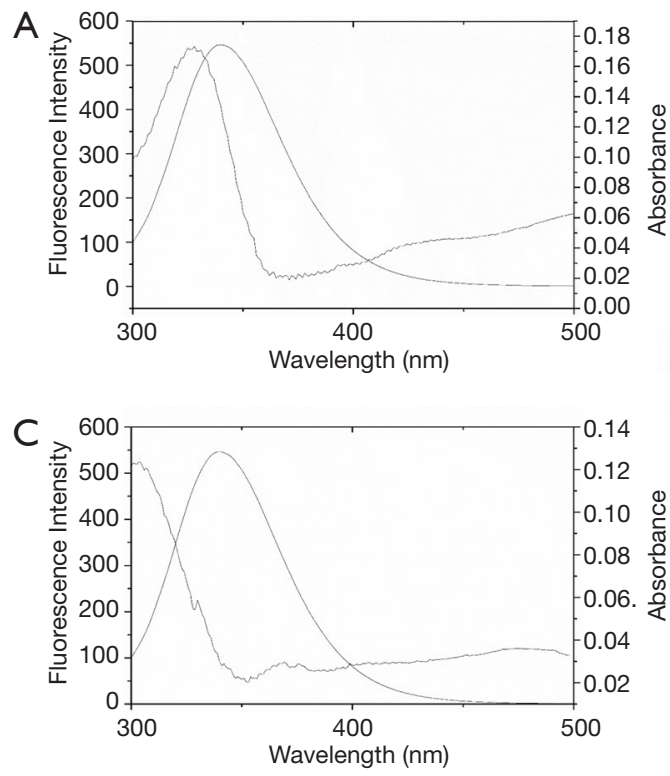

\section{Energy transfer between BSA and haloemodin}

To evaluate the distance between haloemodin and the tryptophan residues in BSA, the overlap of the fluorescence spectrum of BSA with the absorption of the haloemodin at $298 \mathrm{~K}$ were studied (Figure 6). The values of $\mathrm{R} 0$ could be calculated with the values of $\mathrm{K} 2=2 / 3$, $\mathrm{N}=1.336, \varphi=0.15$ (27). The $\mathrm{r}$ values are $2.29,2.27,2.07$, $2.15 \mathrm{~nm}$ for emodin, HEI1, HEI2, HEI3. The distance between Trp 212 residue of BSA and drugs are far lower than $8 \mathrm{~nm}$ indicating the energy transfer from BSA to drugs and the presence of static quenching mechanism (28). Meanwhile, the distance for HEI2-BSA was smaller than that for emodin-BSA, showing a more constant binding process in HEI2-BSA.

\section{Conformational investigation of BSA induced by haloemodin}

To verify the binding mechanism of the haloemodin-BSA complex and the secondary structural changes of BSA, CD studies were carried out on the haloemodin-BSA complex and BSA.

Figure 7 shows the CD spectra of BSA with haloemodin.
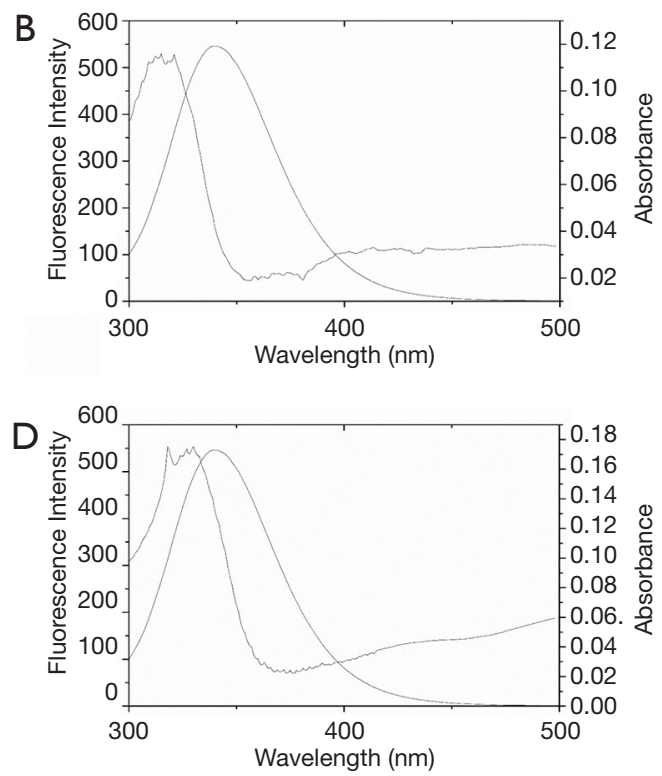

Figure 6 Spectral overlap of emodin and haloemodin absorption spectra with BSA fluorescence. (A) Emodin; (B) HEI1; (C) HEI2; (D) HEI3. BSA =1 $\mu \mathrm{M}$, emodin = HEI1 = HEI2 = HEI3 = $5 \mu \mathrm{M}$. BSA, bovine serum albumin; HEI1, 2-iodioemodin; HEI2, 2,4-diiodoemodin; HEI3, 2,4,5-triiodoemodin. 


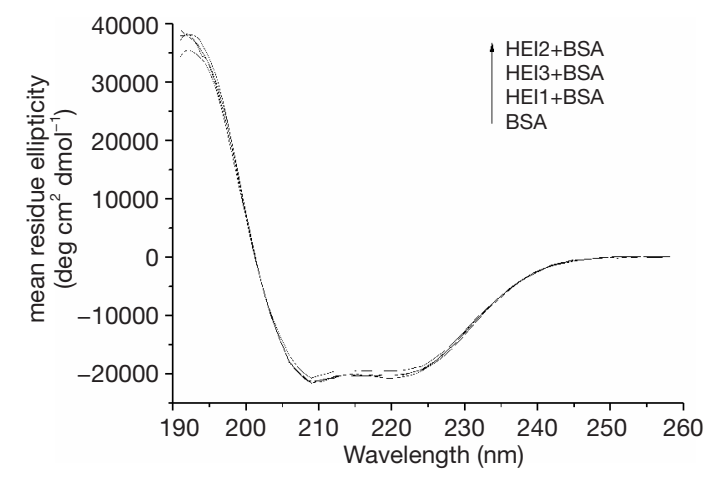

Figure $7 \mathrm{CD}$ spectra of free BSA and its complexation with HEI1, HEI2, and HEI3. BSA = HEI1 = HEI2 = HEI3 $=1 \mu \mathrm{M}$. BSA, bovine serum albumin; HEI1, 2-iodioemodin; HEI2, 2,4-diiodoemodin; HEI3, 2,4,5-triiodoemodin.

Table 4 The contents of $\alpha$-helix in the BSA-haloemodin system

\begin{tabular}{lc}
\hline Experimental samples & The content of $\alpha$-helix (\%) \\
\hline $1 \mu \mathrm{M} \mathrm{BSA}$ & 67.2 \\
$1 \mu \mathrm{M} \mathrm{BSA}+1 \mu \mathrm{M} \mathrm{HEl1}$ & 66.1 \\
$1 \mu \mathrm{M} \mathrm{BSA}+1 \mu \mathrm{M} \mathrm{HEI} 2$ & 61.9 \\
$1 \mu \mathrm{M} \mathrm{BSA}+1 \mu \mathrm{M} \mathrm{HEI3}$ & 64.9 \\
\hline
\end{tabular}

BSA, bovine serum albumin; HEl1, 2-iodioemodin; HEI2, 2,4-diiodoemodin; HEI3, 2,4,5-triiodoemodin.

There are two negative bands (222 and $208 \mathrm{~nm}$ ) in the distant UV region which have a typical $\alpha$-helix protein structure $(29,30)$. These bands resulted from the $n-\pi^{*}$ transition in the peptide bond of $\alpha$-helix. The CD data induced the partial unfolding of the $\alpha$-helix structure. The $\alpha$-helix content of BSA, as calculated by the CD spectra deconvolution software, is presented in Table 4. It can be seen that haloemodin, especially HEI2, could alter the secondary structure of BSA, suggesting that the interaction of haloemodin with BSA can lead to a change in the microenvironment of the protein. This accords with the results acquired via fluorescence spectroscopy.

\section{Discussion}

The haloemodin derived in our lab from the Chinese traditional medicine, emodin, exhibits strong antibacterial activity against Gram-positive bacterial strains, including those generally resistant to drugs, such as MRSA and VRE. Compared with emodin, haloemodin more rapidly increases the bacterial plasma membrane permeability for potassium ions. More importantly, the haloemodin binds more tightly and more stably to the proteins than emodin. These results partially explain the stronger antibacterial activities of haloemodin over their parent nucleus, emodin.

In this study, BSA was used as model protein to investigate the binding mode of haloemodin and emodin with protein. We found that HEI2 binds to BSA and forms HEI2-BSA complexes at Trp-212 within the hydrophobic pocket of subdomain IIA. The thermodynamic parameters suggest that the hydrogen bonds and van der Waals interactions are key actors in the HEI2-BSA binding process, while in the emodin-BSA complex, hydrophobic interactions play the main role. Moreover, the distance between HEI2 and the BSA binding site, Trp 212 (subdomain II) of BSA, is shorter than that of emodin. HEI 2 also reduces the $\alpha$-helix of BSA and changes the secondary protein structure, while emodin induces no influence on the secondary protein structure. These results indicate that HEI2 has a stronger affinity to BSA than emodin, which support our previous findings wherein HEI2 hindered the catalytic activities of DNA gyrase and bacterial topoisomerase I more efficiently than emodin (22). Interestingly, it can be found in most bioactive proteins, such as BSA, E. coli DNA gyrase (GenBank: AHN18133.1), S. aureus DNA gyrase (GenBank: CAA50570.1), and E. coli Topo I (GenBank: KDN05281.1). Thus, we can consider looking at the binding mode of small molecules and BSA as a viable alternative approach to investigating the interaction of small molecules and proteins.

In addition, since BSA is an economical, commercialized, and extensively studied protein, the binding mode study of drugs with proteins using BSA as a model protein can be a novel, practical method for the screening of new drugs and the conducting of pharmacological research.

\section{Acknowledgments}

Funding: This work was financially supported by the National "12th-5-Year" Plan Project of China (2006BAB04A14 and 2011BAJ07B04)" and the National Natural Science Foundation of China (No. 20972105).

\section{Footnote}

Conflicts of Interest: The authors have no conflicts of interest to declare. 
Ethical Statement: The authors are accountable for all aspects of the work in ensuring that questions related to the accuracy or integrity of any part of the work are appropriately investigated and resolved.

\section{References}

1. Wang L, Li D, Bao C, et al. Ultrasonic extraction and separation of anthraquinones from Rheum palmatum L. Ultrason Sonochem 2008;15:738-46.

2. Dai G, Ding K, Cao Q, et al. Emodin suppresses growth and invasion of colorectal cancer cells by inhibiting VEGFR2. Eur J Pharmacol 2019;859:172525.

3. Ahmad E, Sen P, Khan RH. Structural stability as a probe for molecular evolution of homologous albumins studied by spectroscopy and bioinformatics. Cell Biochem Biophys 2011;61:313-25.

4. Xia S, Ni Y, Zhou Q, et al. Emodin Attenuates Severe Acute Pancreatitis via Antioxidant and Anti-inflammatory Activity. Inflammation 2019;42:2129-38.

5. Ji X, Liu X, Peng Y, et al. Comparative analysis of methicillin-sensitive and resistant Staphylococcus aureus exposed to emodin based on proteomic profiling. Biochem Biophys Res Commun 2017;494:318-24.

6. Zhou XM, Chen QH. Biochemical study of Chinese rhubarb. XXII. Inhibitory effect of anthraquinone derivatives on $\mathrm{Na}+-\mathrm{K}+-\mathrm{ATP}$ ase of the rabbit renal medulla and their diuretic action. Yao Xue Xue Bao 1988;23:17.

7. Han W, Sun J, Feng L, et al. Autophagy inhibition enhances daunorubicin-induced apoptosis in K562 cells. PLoS One 2011;6:e28491.

8. Mikusová $\mathrm{V}$, Tichý $\mathrm{A}$, Rezáčová $\mathrm{M}$, et al. Mitoxantrone in combination with a DNA-PK inhibitor: possible therapy of promyelocytic leukaemia resistant forms. Folia Biol (Praha) 2011;57:200-5.

9. Duan F, Li X, Cai S, et al. Haloemodin as novel antibacterial agent inhibiting DNA gyrase and bacterial topoisomerase I. J Med Chem 2014;57:3707-14.

10. Carter DC, Ho JX. Structure of serum albumin. Advances in protein chemistry. Academic Press 1994;45:153-203.

11. Olson RE, Christ DD. Plasma protein binding of drugs. Annual reports in medicinal chemistry. Academic Press 1996;31:327-36.

12. Papadopoulou A, Green RJ, Frazier RA. Interaction of flavonoids with bovine serum albumin: a fluorescence quenching study. J Agric Food Chem 2005;53:158-63.

13. Ghosh KS, Sahoo BK, Dasgupta S.
Spectrophotometric studies on the interaction between (-)-epigallocatechin gallate and lysozyme. Chem Phys Lett 2008;452:193-7.

14. Eftink MR, Ghiron C. Fluorescence quenching studies with proteins. Anal Biochem 1981;114:199-227.

15. Lakowicz JR, Weber G. Quenching of fluorescence by oxygen. Probe for structural fluctuations in macromolecules. Biochemistry 1973;12:4161-70.

16. Xie MX, Xu XY, Wang YD. Interaction between hesperetin and human serum albumin revealed by spectroscopic methods. Biochim Biophys Acta 2005;1724:215-24.

17. Förster T. 10th Spiers Memorial Lecture. Transfer mechanisms of electronic excitation. Discussions of the Faraday Society 1959;27:7-17.

18. Sun W, Jiao K. Linear sweep voltammetric determination of protein based on its interaction with Alizarin Red S. Talanta 2002;56:1073-80.

19. Heller DP, Greenstock CL. Fluorescence lifetime analysis of DNA intercalated ethidium bromide and quenching by free dye. Biophys Chem 1994;50:305-12.

20. Cui FL, Fan J, Ma DL, et al. A study of the interaction between a new reagent and serum albumin by fluorescence spectroscopy. Anal Lett 2003;36:2151-66.

21. Förster T. Delocalized Excitation and Excitation Transfer. Florida, Tallahassee: Florida State University, 1965:Bulletin (18).

22. Albrecht C, Lakowicz JR: Principles of fluorescence spectroscopy. Anal Bioanal Chem 2008;390:1223-24.

23. Tang J, Luan F, Chen X. Binding analysis of glycyrrhetinic acid to human serum albumin: fluorescence spectroscopy, FTIR, and molecular modeling. Bioorg Med Chem 2006;14:3210-17.

24. Pace C N, Vajdos F, Fee L, et al. How to measure and predict the molar absorption coefficient of a protein. Protein Sci 1995;4:2411-23.

25. Ross PD, Subramanian S. Thermodynamics of protein association reactions: forces contributing to stability. Biochemistry 1981;20:3096-102.

26. Tian J, Liu J, Hu Z, et al. Interaction of wogonin with bovine serum albumin. Bioorg Med Chem 2005;13:4124-9.

27. Miller JN. Recent advances in molecular luminescence analysis. Proc Anal Div Chem Soc 1979;16:203-8.

28. Lu Y, Cui F, Fan J, et al. Interaction of human serum albumin with $\mathrm{N}$-(4-ethoxyphenyl)-N'-(4-antipyrinyl) thiourea using spectroscopies and molecular modeling method. J Lumines 2009;129:734-40. 
29. Norde W, Giacomelli CE. BSA structural changes during homomolecular exchange between the adsorbed and the dissolved states. J Biotechnol 2000;79:259-68.

Cite this article as: Ji C, Xin G, Duan F, Huang W, Tan T. Study on the antibacterial activities of emodin derivatives against clinical drug-resistant bacterial strains and their interaction with proteins. Ann Transl Med 2020;8(4):92. doi: 10.21037/atm.2019.12.100
30. Greenfield N, Fasman GD. Computed circular dichroism spectra for the evaluation of protein conformation. Biochemistry 1969;8:4108-16. 